\title{
Histoire et développement des sources de rayonnement synchrotron
}

L'histoire du rayonnement synchrotron (noté RS par la suite), même si elle semble toute récente avec le développement de son utilisation pour l'étude de la matière depuis trente ans, remonte cependant à la fin du siècle dernier. Les travaux de Liénard, Wiechert et Schott sur les particules électriquement chargées soumises à une accélération, ont montré qu'elles rayonnent un champ électromagnétique.

$C^{\prime}$ est le cas des électrons impactant l'anode dans un tube à rayons $X$ : ainsi ralentis, ils émettent un rayonnement dit de freinage ("bremsstrahlung"). Par contre, il sera usuellement question de RS lorsque la particule chargée électriquement est relativiste et rayonne sous l'effet d'un champ magnétique transverse à sa trajectoire.

C'est au début des années 1940 que le RS a été étudié en Russie [1] et aux USA [2]. Un nouveau type d'accélérateur, nommé synchrotron, a ainsi vu le jour à la General Electric (USA), conduisant à la première observation du RS dans le domaine du visible [3]. La théorie classique relativiste de cette émission a été formulée peu après par Schwinger [4].

Le début des années 1950 a vu la première ligne de RS, dans le domaine ultraviolet à vide, à Cornell (États-Unis). Tomboulian et Hartman [5] ont ainsi mis en évidence l'émission d'un faisceau blanc, caractéristique du RS pour une trajectoire circulaire des électrons. Il a fallu attendre ensuite les années 1960 pour que de nouvelles études voient le jour auprès de synchrotrons dédiés à la physique des hautes énergies, comme ACO à Orsay, suivi sur ce même site par DCI (1976) puis SuperACO (1987). Parmi les premières utilisations du RS, citons aux États-Unis des études sur la polarisation à Cornell [6], sur l'absorption par les gaz au NBS à Washington [7] et, en Europe, les travaux pionniers de l'équipe d'Y. Cauchois $[8,9]$ réalisés à Frascati en Italie. La figure 1 montre l'anneau de Frascati.

C'est seulement dans les années 1970 qu'une utilisation plus intense du RS est apparue, notamment en Europe, à Hambourg (Allemagne), Daresbury (Angleterre) et Orsay (France).

L'utilisation du RS, qui n'était produit que de façon parasite sur les accélérateurs de physique des hautes énergies, s'est considérablement développée vers le milieu des années 1980 sur des machines dédiées : il s'agit d'anneaux de stockage, dans lesquels la vitesse des électrons est maintenue constante. Le RS émis y est issu soit des aimants de courbure assurant la fermeture de la trajectoire sur elle-même, soit de dispositifs insérés entre ces aimants; le spectre est blanc dans le premier cas, alors qu'on observe un spectre de raies dans le second.

Par son intensité et sa qualité, sans commune mesure avec les rayons X classiques, le RS intéresse pratiquement toutes les disciplines : physique, chimie, biologie et médecine, sciences de la Terre et de l'univers, paléontologie, métrologie, nanomonde, etc. De là résulte son développement considérable depuis trente ans, d'autant que le progrès des sources a été fulgurant. La figure 2 présente l'accroissement gigantesque de la brillance (le paramètre principal des sources) dans le domaine des rayons $\mathrm{X}$ au cours des années.
On distingue plusieurs étapes de développement, dénotées "générations ". La première est l'utilisation en parasite sur les accélérateurs de particules de physique des hautes énergies, la seconde correspondant aux premières machines circulaires dédiées au RS émis par les aimants de courbure. La troisième est axée sur l'utilisation des dispositifs placés en insertion dans les sections droites (" onduleurs ") ; c'est celle, désormais arrivée à maturité, qui est exploitée dans les articles scientifiques de cette revue. La quatrième génération, celle des Lasers à Électrons Libres (LEL) - dont l'utilisation dans le domaine des rayons $\mathrm{X}$ durs a juste débuté [10] - fait intervenir des accélérateurs linéaires pour produire dans des onduleurs des bouffées ultracourtes de rayonnement totalement cohérent. Enfin, on peut parler de sources de cinquième génération avec les accélérateurs à plasma laser, qui sont au stade de la recherche [11] et de début de réalisation [12].

Il est très important de noter que les deux dernières générations, qui apporteront principalement l'accès aux temps ultracourts (femto- et attosecondes !) ne supplanteront pas les machines circulaires de $3^{e}$ génération, qui présentent encore des marges importantes de progrès, tant au niveau de la source elle-même que de son utilisation. Il ne faut en effet pas perdre de vue que toute expérience dépend de l'optimisation de l'ensemble de la ligne de lumière : après la source, l'optique du filtrage géométrique et spectral du faisceau de photons émis par cette source, puis plus en aval l'environnement et le positionnement de l'échantillon analysé et enfin, et ce n'est pas le moindre des aspects, la détection des photons et électrons résultant de l'interaction. Cette optimisation, encore en développement sur la $3^{\text {e }}$ génération, prendra longtemps pour la $4^{e}$ génération, qui détruit généralement par sa puissance la cible d'étude, et dont la cohérence n'est pas toujours nécessaire quand elle n'est pas nuisible. C'est aussi le cas de la $5^{e}$ génération, qui en est au stade initial de la recherche, et dont les caractéristiques finales pour son exploitation sont par conséquent inconnues. De plus, le spectre d'émission atteint difficilement les rayons $\mathrm{X}$ durs pour la $4^{e}$, et la $5^{e}$ pourrait être limitée, pour les très hautes brillances, aux rayons $\mathrm{X}$ mous. Enfin, les machines 


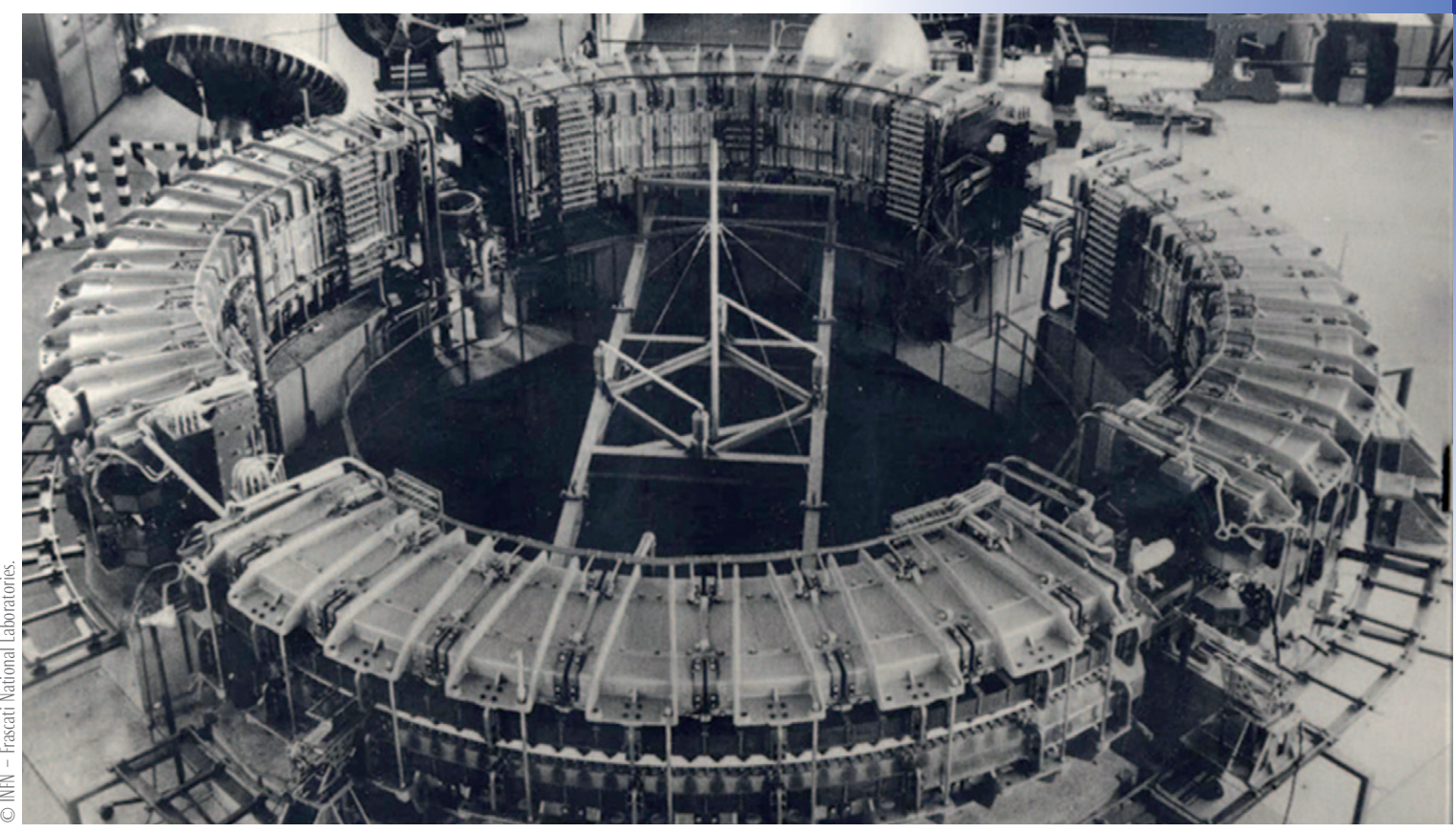

1. Le synchrotron à électrons de Frascati, près de Rome (Italie), pendant une mesure de champ magnétique, lors de sa construction en 1958.

linéaires ne permettent qu'un nombre limité d'utilisateurs, lesquels sont au contraire nombreux sur les machines circulaires.

Il y a actuellement plus de 70 installations dans le monde, offrant le RS sur des accélérateurs circulaires et linéaires (dont

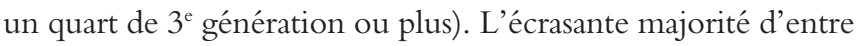
elles, environ $98 \%$, sont situées en Europe (1/3), Asie (1/3) et États-Unis (un peu moins de 1/3), réparties géographiquement autour du $45^{\mathrm{e}}$ parallèle. Une liste des principales sources RS de $3^{\mathrm{e}}$ génération est donnée dans le tableau 1.

En France, se trouvent aujourd'hui deux laboratoires de $3^{\text {e }}$ génération, SOLEIL et l'ESRF, de statuts différents. L'ESRF est l'unique installation au monde qui soit internationale (en l'occurrence européenne), car construite et cogérée, à l'instar du CERN, par plusieurs pays (dix-neuf aujourd'hui). SOLEIL, de son côté, est un équipement national, même s'il est ouvert plus largement. Une autre différence est la date d'ouverture aux utilisateurs, 1994 pour l'ESRF, quinze ans plus tard pour SOLEIL. Le fait d'avoir ces deux grandes installations sur le sol français est une source d'enrichissement mutuel, scientifique et technologique. Sur le plan des photons délivrés, SOLEIL couvre tout le spectre jusqu'aux rayons X, l'ESRF étant optimisé pour les rayons $\mathrm{X}$ durs, en raison de la différence d'énergie des électrons circulant dans ces deux anneaux (respectivement $2,75$ et $6 \mathrm{GeV})$.

Michel Belakhovsky Ancien physicien CEA et ESRF

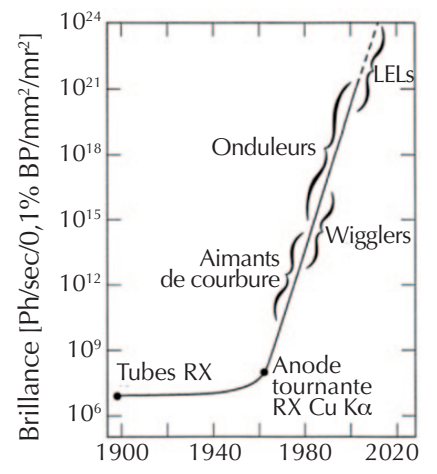

\section{Références}

1• I. Ya. Pomeranchuk, Sov. Phys. JETP 9 (1939) 915.

2• E.M. McMillan, Phys. Rev. 68 (1945) 144.

3• F.R. Elder, A.M. Gurewitsch, R.V. Langmuir et H.C. Pollock, Phys. Rev. 71 (1947) 829.

4• J. Schwinger, Phys. Rev. 75 (1949) 1912.

5• D.H. Tomboulian et P.L. Hartman, Phys. Rev. 102 (1956) 1423.

6• P. Joos, Phys. Rev. Lett. 4 (1960) 558.

7• R.P. Madden et K. Codling, Phys. Rev. Lett. 10 (1963) 516.

8• P. Jaegle, Synchrotron Radiation News, 2 (1989) 22-23.

9• Y. Cauchois, C. Bonnelle et G. Missoni, C.R. Acad. Sci. Fr. 257 (1963) 409 et 1242. 10• Aux États-Unis, le LCLS : https://portal.slac.stanford.edu/sites/Icls_public/ Pages/Default.aspx

Le X-FEL européen devrait entrer en opération en 2015 : www.xfel.eu/

11• D. Hulin, Reflets de la Physique, 24 (2011) 4-8.

12• V. Malka et al., Reflets de la Physique, 33 (2013) 23-26.

Anneaux de stockage de $3^{\text {e }}$ génération pour le rayonnement synchrotron en rayons $\mathrm{X}$ mous et durs.

\begin{tabular}{|c|c|c|c|c|c|}
\hline Nom & Lieu & Pays & $\begin{array}{c}\text { Énergie } \\
(\mathbf{G e v})\end{array}$ & $\begin{array}{c}\text { Circonférence } \\
\text { (m) }\end{array}$ & $\begin{array}{c}\text { Année de mise } \\
\text { en service }\end{array}$ \\
\hline ESRF & Grenoble & France & 6 & 844 & 1992 \\
\hline Advanced Light Source (ALS) & Lawrence Berkeley Laboratory & USA & 1,9 & 196,8 & 1993 \\
\hline ELETTRA & Trieste & Italie & 2,0 et 2,4 & 260 & 1993 \\
\hline Advanced Photon Source (APS) & Argonne National Laboratory & USA & 7,0 & 1104 & 1995 \\
\hline Spring-8 & RIKEN, Kobe & Japon & 8 & 1436 & 1997 \\
Bessy II & Berlin & Allemagne & 1,7 & 240 & 1998 \\
\hline Swiss Light Source & PSI, Villigen & Suisse & 2,8 & 288 & 2001 \\
SOLEIL & Saclay & France & 2,75 & 354 & 2006 \\
\hline Diamond Light Source & Harwell (Oxfordshire) & UK & 3 & 561,8 & 2007 \\
\hline PETRA III & DESY, Hambourg & Allemagne & 6,5 & 2304 & 2009 \\
\hline
\end{tabular}

